\title{
Performance Analysis \\ of Dynamic OFDMA Systems \\ with Inband Signaling
}

\author{
James Gross $\diamond$, Hans-Florian Geerdes^, Holger Karl», Adam Wolisz ${ }^{\diamond}$ \\ $\diamond$ Telecommunication Networks Group, Technische Universitaet Berlin \\ Einsteinufer 25, 10587 Berlin, Germany \\ Phone: +49 30 31423830, E-mail: \{gross,wolisz\}@ tkn.tu-berlin.de \\ *Zuse Institute Berlin (ZIB) \\ Takustr. 7, 14195 Berlin, Germany \\ Phone: +49 30 84185278, E-mail: geerdes@zib.de \\ -Institute of Computer Science, University of Paderborn \\ Phone: +49 525160 3002, E-mail: holger.karl@uni-paderborn.de
}

This work has been supported partially by the German research funding agency 'Deutsche Forschungsgemeinschaft (DFG)' under the graduate program 'Graduiertenkolleg 621 (MAGSI/Berlin)' and by the DFG Research Center MATHEON "Mathematics for key technologies" in Berlin.

This work has been done while Holger Karl was with the Telecommunication Networks Group at the TU Berlin. 


\title{
Index Terms
}

Multi-user OFDM, optimization, inband signaling, dynamic OFDMA

\begin{abstract}
Within the last decade the OFDM transmission scheme has become part of several standards for wireless systems. Today, OFDM is even a candidate for $4 \mathrm{G}$ wireless systems. It is well known that dynamic OFDMA systems potentially increase the spectral efficiency. They exploit diversity effects in time, space, and frequency by assigning system resources periodically to terminals. Informing the terminals about new assignments creates a signaling overhead. Up to now, this overhead has not been taken into account in studies on dynamic OFDMA systems. Yet this is crucial for a realistic notion of the performance achieved by dynamic approaches. In this paper, we close this gap. We introduce two forms of representing the signaling information and discuss how these affect system performance. The study of the signaling impact on the performance is conducted for an exemplary dynamic approach.

We find that the throughput behavior of dynamic OFDMA systems is significantly influenced by the signaling overhead. In many situations, neglecting the overhead leads to wrong performance conclusions. Also, the performance difference between dynamic and static schemes is now much more sensible to the specific parameter set of the transmission scenario (e.g. frame length, sub-carrier number etc.). This leads to the proposal of access points which should adapt certain system parameters in order to provide optimal performance.
\end{abstract}

\section{INTRODUCTION}

Orthogonal Frequency Division Multiplexing (OFDM) appears to be quite an attractive transmission scheme in order to overcome the impairments of wireless broadband channels. In OFDM systems the total bandwidth is split into many subchannels, also referred to as sub-carriers [1], [2]. This reduces the symbol length per sub-carrier leading to a much lower sensitivity to Intersymbol Interference (ISI) . Due to this fact, various standards for wireless systems are meanwhile based on the OFDM transmission scheme. OFDM is also a strong candidate as transmission scheme for future cellular wideband communications systems (e.g. 4G systems).

From a link layer perspective, the principal advantage of OFDM in frequency-selective channels is accompanied by certain (cross-layer) optimization opportunities. These arise due to strong diversity in frequency, time, and space. Considering the down-link transmission direction, the attenuation of different sub-carriers supporting a certain terminal usually varies strongly (due to frequency-selective fading); furthermore, the attenuation of the same sub-carrier varies for 
different terminals (due to multi-user diversity). Finally, the attenuations vary over time. It is well known that this high degree of variation can be exploited by appropriately constructed dynamic link layers.

The concept of a dynamic link layer for OFDM systems is quite simple. Assume that the transmitter (the access point) knows the current sub-carrier attenuations between access point and terminals; furthermore, consider the attenuations to be constant for some time span. Then at least two forms of dynamic adaption are possible: dynamic sub-carrier assignment and dynamic power assignment. As discussed above, the attenuation for a sub-carrier differs strongly for different terminals. Therefore, a better spectrum utilization can be achieved by assigning (selected) disjunctive sets of sub-carriers to the terminals. These assignments are re-allocated periodically in order to adapt to the time-varying channel attenuations. This approach in multiuser OFDM systems is referred to as dynamic Orthogonal Frequency Division Multiple Access (OFDMA) [3], [4], [5]. The dynamic adaption can be used either to increase the throughput of a system or to decrease the transmit power.

The second parameter that can be adapted is the transmission power used on a subcarrier. Dynamically distributing the transmit power can lead to a further improvement of the performance. However, the computational requirements for these schemes are higher. Much attention has been spent on the complexity of such dynamic OFDMA schemes, as the real-time requirements are quite demanding given that the coherence time of a wireless channel is in the range of a few milliseconds.

However, there is an overhead related to dynamic OFDMA systems. The access point generates new assignments for payload transmission periodically (i.e. for each down-link phase). The receiving terminals need to know the assignment information in order to decode and recombine the transmitted payload correctly. Therefore, a signaling phase in which the assignment information is transmitted to the terminals has to be introduced prior to payload transmission. This certainly affects the performance of a dynamic OFDMA system.

Investigations considering dynamic OFDMA systems usually ignore this overhead. We are not aware of any study addressing this overhead. Only in the context of bit-loading (dynamic adaption for point-to-point connections) two related studies have been published [6], [7]. However, these studies do not consider the principal performance impact due to the signaling overhead. Instead, they focus on suitable compression schemes.

For multi-user OFDMA systems, no such study is known. In this paper we present a systematic investigation on modeling the signaling overhead and optimizing the performance 
of the resulting model.

The performance gain achieved by dynamically assigning sub-carriers can be significantly increased by minimizing the signaling overhead. The main idea is to choose new assignments with knowledge of the previous assignments, and to change assignments only if the gain in throughput is larger than the signaling overhead caused by the reassignment. This leads to a difficult quadratic optimization problem for which we propose a novel algorithm. We compare the achieved performance of this novel scheme to two computationally "cheaper" variants, which have been already presented in previous work [8], [9]. The performance comparison is done for a wide range of parameters, revealing the fact that including the signaling overhead leads to a new qualitative behavior of dynamic OFDMA systems. For example, increasing the number of sub-carriers for a given bandwidth increases the average throughput as such. But it also causes a higher signaling cost, reducing the overall performance again. We show that this trade-off leads to an optimal choice of the number of sub-carriers the bandwidth should be split into. This result could be used by base stations for dynamic OFDMA systems, which adapt system parameters in order to provide optimal performance.

The remainder of the paper is organized as follows: In Section II we present our system model. We formulate the basic dynamic OFDMA approach in Section III and discuss in Section IV several methods to incorporate the signaling overhead into the optimization model. Section $\mathrm{V}$ compares the performance of the three approaches in a computational study. In Section VI we draw conclusions and give an outlook on future work.

\section{SYSTEM MODEL}

We consider the down-link of a single cell of a wireless system with radius $r_{\text {cell }}$. The access point serves $J$ terminals by sending a continuous stream of data to each of them. The terminals are uniformly distributed over the area of the cell. We consider a slotted system in which time is divided into units (frames) of duration $T_{\mathrm{f}}$.

A total bandwidth of $B[\mathrm{~Hz}]$ at the center frequency $f_{\mathrm{c}}$ is available for data transmission. For this band, a maximum total transmit power of $P_{\max }$ may be emitted. The given bandwidth is split into $N$ sub-carriers. Although the symbol duration $\frac{N}{B}=T_{\mathrm{s}}$ is equal for each sub-carrier, a different amount of bits might be represented per symbol for each sub-carrier. A total of $M$ modulation types are available. One OFDM symbol is generated per symbol time. Prior to transmitting each OFDM symbol the guard interval of duration $T_{\mathrm{g}}$ is added.

The perceived signal quality per sub-carrier, i.e. the SNR, varies from frame to frame. The 
instant SNR of sub-carrier $n$ for terminal $j$ at time $t$ is given by $v_{j, n}^{(t)}=\frac{p_{n}^{(t)} \cdot\left(h_{j, n}^{(t)}\right)^{2}}{\sigma^{2}}$. Here $p_{n}^{(t)}$ denotes the transmission power, $h_{j, n}^{(t)}$ denotes the attenuation of sub-carrier $n$, and $\sigma^{2}$ is the noise power. The attenuation is responsible for the variation of the perceived SNR; it varies due to path loss, shadowing and fading ${ }^{1}$. Thus, $h_{j, n}^{(t)}$ can be decomposed into three factors modeling these three effects (yielding $h_{,}^{(t)}=h_{\mathrm{pl}}^{(t)} \cdot h_{\mathrm{sh}}^{(t)} \cdot h_{\text {fad }}^{(t)}$ ). The attenuation of each subcarrier is assumed to be constant per frame of duration $T_{\mathrm{f}}$. Note that this duration is considered to be shorter than the coherence time [10] of the sub-carriers.

Each frame is split into a down-link and an up-link phase (Figure 1). OFDMA is applied during the down-link phase. Thus, we consider an OFDM-TDD system here. The duration of one down-link phase (including the signaling information) is denoted by $T_{\mathrm{d}}$. Prior to each down-link phase, the access point generates new assignments of sub-carriers to terminals. Delay effects due to an inadequate processing power at the access point are not considered. The terminals need to be informed of the new assignments in advance. A certain time span of each down-link phase is therefore used to transmit the signaling information. This time span is called the signaling phase. We consider an inband signaling scheme: The signaling information is transmitted in the bandwidth that is also used for payload data transmission ${ }^{2}$. In addition, we assume the signaling information to be broadcasted during the signaling phase using a predefined, fixed modulation type, denoted by $b_{\text {sig. }}$. Broadcasting the signaling information adds to the system's flexibility; terminals can easily receive arbitrary numbers of sub-carriers without loosing synchronization to the dynamic assignments.

The generation of the new assignments per frame is based on the knowledge of the (future) sub-carrier states, denoted by matrix $\mathbf{H}^{(t)}=\left(h_{j, n}^{(t)} \mid \forall j, n\right)$. We assume perfect channel knowledge at the access point. Such an channel knowledge can be achieved closely, if the terminals measure sub-carrier attenuations during a down-link phase and transmit the results to the access point in the next up-link phase. This, again, creates an overhead during the up-link phase. In the present work we focus on the signaling overhead. However, some remarks on the up-link load can be found in Section V-C.

\footnotetext{
${ }^{1}$ In this study the terminals are assumed to be stationary, thus the time-selective fading is caused by moving objects within the propagation environment. However, for the time-selective fading (and thus for the correlation in time) one could also assume the terminals to be mobile

${ }^{2}$ In some studies regarding dynamic OFDMA the signaling information is assumed to be transmitted by a separate control channel. However, none of these studies quantified the bandwidth requirements for such a channel.
} 


\section{DYNAMIC OFDMA APPROACH}

For each down-link phase $t$, the access point assigns sub-carriers to terminals. In the following, we represent the assignment decision with binary variables $x_{j, n}^{(t)}(=1$ if terminal $j$ is assigned sub-carrier $n$ for down-link phase $t ;=0$ otherwise).

All binary assignment variables for down-link phase $t$ are grouped in the assignment matrix $\mathbf{X}^{(t)}=\left(x_{j, n}^{(t)} \mid \forall j, n\right)$. Each sub-carrier can be assigned to at most one terminal at a time. This yields a linear constraint:

$$
\sum_{j} x_{j, n}^{(t)} \leq 1 \quad \forall n
$$

The sub-carrier attenuations are denoted by $h_{j, n}^{(t)}$. We assume perfect sub-carrier attenuation knowledge at the access point.

In this study, we focus on maximizing the system throughput by assigning sub-carriers dynamically. We do not consider additional power adaption, since this requires even more computational power at the access point. We rather assume that the transmit power is evenly spread on the sub-carriers, each one receiving a power of $p_{n}^{(t)}=\frac{P_{\max }}{N}$. The SNR for different subcarriers thus varies according to the attenuations. These variations are exploited using adaptive modulation.

The adaptive modulation scheme works as follows: Given the transmission power per subcarrier, the noise power and the actual attenuation $h_{j, n}^{(t)}$ per terminal $j$, the SNR can be computed that would be reached if sub-carrier $n$ is assigned to terminal $j$ during the next down-link phase. Depending on this SNR value, the modulation type with the highest number of bits transmitted per symbol is chosen (out of the $M$ possible modulation types) with respect to some predefined target symbol error probability $p_{\mathrm{sym}, \max }$. This way the matrix consisting of the attenuations $h_{j, n}^{(t)}$ can be converted into a bit matrix with values $b_{j, n}^{(t)}$. The bit matrix denotes the potential amount of bits, which can be transmitted to each terminal per OFDM symbol on each subcarrier if this pair of sub-carrier and terminal is chosen as assignment. For the specific relation between SNR value and chosen modulation type assumed in this paper, refer to Section V-B.

The total number of symbols per down-link phase is denoted by $S=\left\lfloor T_{\mathrm{d}} /\left(T_{\mathrm{s}}+T_{\mathrm{g}}\right)\right\rfloor^{3}$. Given a certain assignment realization $\mathbf{X}^{(t)}$, the total number of bits that can be transmitted in

\footnotetext{
${ }^{3}$ In a real system layout, these durations are chosen such that the division yields an integer value. However, in the studied system scenarios sometimes the parameters did not result in an integer number. In these cases we chose the floor.
} 
the entire down-link phase $t$ is given by

$$
S \sum_{j, n} b_{j, n}^{(t)} \cdot x_{j, n}^{(t)} .
$$

This overall data amount per down-link phase serves as our metric, the objective is to maximize this value. However, this is a gross value as it does not contain the performance loss due to signaling.

For avoiding starvation of terminals far away from the access point, each terminal is allocated a certain amount of sub-carriers. We follow here a two-step approach [11], [12] where first sub-carriers are allocated (determining the number of sub-carriers each terminal receives) and are then assigned (determining which sub-carriers each terminal receives). We do so by fixing a maximum number $l_{j}$ of sub-carriers that each terminal $j$ can obtain. This can be formulated as a linear constraint:

$$
\sum_{n} x_{j, n}^{(t)} \leq l_{j} \quad \forall j
$$

Based on the notation introduced, the maximization of gross throughput in the dynamic OFDMA system can be formulated as a linear integer programming optimization problem, referred to as (PLAIN):

$$
\max \quad S \cdot \sum_{j, n} b_{j, n}^{(t)} \cdot x_{j, n}^{(t)} \quad \text { s.t. } \quad(1),(3) .
$$

In graph theory, (PLAIN) is called the bipartite weighted b-matching problem. In the present case, it can be shown that it has a complexity of at most $O\left(N^{3}\right)$ using regular matching algorithms [13]. In practice, the optimal solutions to this problem can be computed within milliseconds [14] for the problem sizes considered here using linear programming algorithms, cf. the remarks in V-A.

\section{SignALING SCHEMES AND THEIR IMPLEMENTATION}

In this section, we discuss two different models to represent the signaling information. These signaling models are discussed in the context of the presented dynamic OFDMA approach (PLAIN). However, they can also be used to convey the signaling information of any other dynamic OFDMA approach (for example, the ones presented in [4], [3]).

Assume some algorithm has generated the solution of an instance of (PLAIN) at the access point. The assignment information is contained in the matrix $\mathbf{X}^{(t)}$. In addition, the applied modulation type has to be signaled for each sub-carrier assignment. Therefore, the basic information unit of the signaling information consists of the triple: 〈sub-carrier identification, terminal address, 
modulation identification $\rangle$. These units can be transmitted to the terminals in - at least - two different ways. We will call the first, straightforward approach the fixed size signaling field model. All assignments are transmitted, regardless of whether or not an assignment has changed from the previous to the current down-link phase. The second approach is called the variable size signaling field model. Here, only the assignment changes from one down-link phase to the next one are conveyed. As a consequence, a varying number of assignments are transmitted to the terminals.

\section{A. "Fixed Size Signaling Field” Approach}

We propose for this approach the following procedure [8] (Figure 2(a)): All $N$ assignments are transmitted for each down-link phase. The position of the tuple 〈terminal address, modulation identification〉 in the signaling bit sequence indicates the subcarrier identification. Therefore, a cost of $\left\lceil\log _{2}(J)\right\rceil+\left\lceil\log _{2}(M)\right\rceil$ bits applies per assignment. Transmitting all assignments results in a total cost (in bits) of $N \cdot\left(\left\lceil\log _{2}(J)\right\rceil+\left\lceil\log _{2}(M)\right\rceil\right)$, which requires a total of

$$
\varsigma=\left\lceil\frac{\left(\left\lceil\log _{2}(J)\right\rceil+\left\lceil\log _{2}(M)\right\rceil\right)}{b_{\text {sig }}}\right\rceil
$$

symbols per down-link phase (note that all $N$ sub-carriers can be used for transmission). Consequently, $S-\varsigma$ symbols remain for payload transmission. Inserting this expression (instead of only $S$ ) in Equation (2) yields the effective, net throughput.

Since the number of lost symbols due to signaling is constant for a fixed system instance (parameter set), maximizing the net throughput is equivalent to maximizing the gross throughput of (PLAIN) (using the same efficient algorithm). However, the number of symbols available for payload transmission is smaller.

$$
\max \quad(S-\varsigma) \cdot \sum_{j, n} b_{j, n}^{(t)} \cdot x_{j, n}^{(t)} \quad \text { s.t. } \quad(1),(3) .
$$

\section{B. "Variable Size Signaling Field" Approach - Optimal Model}

In order to save overhead, it is possible to transmit only the assignments of sub-carriers that have changed with respect to the last down-link phase. As a consequence, the binary representation of one assignment becomes more expensive. In order to identify a sub-carrier, $\left\lceil\log _{2}(N)\right\rceil$ bits are required in addition. As with the fixed size signaling field model, the terminal address and modulation identification will consume $\left\lceil\log _{2}(J)\right\rceil$ and $\left\lceil\log _{2}(M)\right\rceil$ bits. In total, transmitting an assignment change from one down-link phase to the next one then consumes 
$C_{\text {sig }}=\left\lceil\log _{2}(N)\right\rceil+\left\lceil\log _{2}(J)\right\rceil+\left\lceil\log _{2}(M)\right\rceil$ bits $^{4}$. The layout of the resulting signaling field is shown in Figure 2. Per assignment, the bit cost is increased by $\left\lceil\log _{2}(N)\right\rceil$ bits. However, if only a few assignments change, the overall cost for signaling in bits is smaller. There is a clear trade-off between exploiting diversity by dynamically reassigning sub-carriers and avoiding reassignments in order to reduce signaling overhead. We propose a state-aware scheme for the access point to decide which dynamic reassignments pay out. Knowing the assignments $\mathbf{X}^{(t-1)}$ for down-link phase $t-1$, the cost for assigning sub-carrier $n$ to terminal $j$ in phase $t$ is $c_{j, n}^{(t)}=C_{\text {sig }}$ if $x_{j, n}^{(t-1)}=0$ and $c_{j, n}^{(t)}=0$ otherwise. During the signaling phase, a fixed modulation type $\left(b_{\mathrm{sig}}\right)$ is used. What has to be maximized is the net throughput of the remaining down-link phase after transmitting $\varsigma=\left\lceil\sum_{j, n} c_{j, n}^{(t)} \cdot x_{j, n}^{(t)} / N \cdot b_{\mathrm{sig}}\right\rceil$ symbols in order to convey the signaling information. This yields the following optimization problem:

$$
\max \left(S-\left\lceil\frac{\sum_{j, n} c_{j, n}^{(t)} \cdot x_{j, n}^{(t)}}{N \cdot b_{\text {sig }}}\right\rceil\right) \cdot \sum_{j, n}\left(b_{j, n}^{(t)} \cdot x_{j, n}^{(t)}\right) \quad \text { s.t. } \quad \text { (1), (3) . }
$$

(VAR OPT)

This problem is nonlinear, our solution approach is described in the appendix. Compared to (FIX), the key difference is that $\varsigma$ is a variable depending on the number of new assignments.

\section{C. "Variable Size Signaling Field" Approach - Approximation Model}

Given an assignment of the previous down-link phase, the solution to (VAR OPT) is optimal for the variable size signaling field model. Solving (VAR OPT) to optimality, however, is computationally difficult due to the problem's quadratic nature. In order to reduce the complexity, we suggest in [9] to consider as optimization objective the difference between the throughput achieved by the current assignment choice and the signaling bit cost, caused by changing some assignments. This neglects the influence of the uniform modulation type during the signaling phase (and so neglects the influence of the variable number of symbols required for signaling).

The number of raw bits transmitted (using a certain assignment) is given by (2). Combining this with $\sum_{j, n} c_{j, n}^{(t)} \cdot x_{j, n}^{(t)}$, the number of signaling bits needed to represent the changed assignments, we obtain the following optimization problem:

$$
\max \quad \sum_{j, n}\left(S \cdot b_{j, n}^{(t)}-c_{j, n}^{(t)}\right) \cdot x_{j, n}^{(t)} \quad \text { s.t. } \quad(1),(3) .
$$

Note that (VAR APP) is equivalent to (FIX) with a modified bit matrix to account for the cost caused by changing assignments. The objective function value is not the net throughput. Given

\footnotetext{
${ }^{4}$ It may happen that a certain sub-carrier is not used at all during the next down-link phase. In this case an identification for the "null"-modulation is required to indicate the assignment change. This leads to an $M$ that is increased by one.
} 
the solution to an instance of (VAR APP), the throughput has to be computed separately by determining the number of required signaling symbols $\varsigma$.

\section{Performance Evaluation}

In this section, we study the performance of the signaling models. At first we introduce our methodology, then we discuss the scenario parameters and finally present the results.

\section{A. Methodology}

From a system point of view, metrics like the average data rate per terminal are of interest. However, the solution for an instance of one of the discussed problems is a certain number of bits that can be transmitted per down-link phase to each terminal. In order to obtain system level results, the following procedure was conducted. Initially, channel trace files of the attenuations of each sub-carrier were generated from a computer program. Each attenuation sample consisted of path loss, shadowing and fading components, as discussed and parameterized in Section V-B. Modeling the correlation of the fading process in time and frequency is crucial. We implemented the fading process in the trace file generator by applying the method of Rice (superposition of appropriately parameterized harmonic functions in order to model a colored Gaussian random process) [15]. Using this method for the fading, one sample was generated for every down-link phase and sub-carrier per terminal. Once the trace file of the attenuations was generated, the attenuation matrix was transformed into the bit matrix $\mathbf{B}^{(t)}$ for each down-link phase.

Specialized algorithms from graph theory are known for problems (FIX) and (VAR APP), for a survey see [13]. It is therefore not necessary to employ integer programming algorithms. However, state-of-the-art matching algorithms are complex to implement and might therefore not be the method of choice in a real system. On the other hand, due to the special properties of the so-called $b$-matching polytope [16], the problems can be solved with linear programming algorithms and the resulting solutions will always be integral. We have thus decided to use linear programming software for the results in this paper, leaving the question of what assignment algorithms and possibly heuristics to use in a real system for future work.

For problem (VAR OPT) we propose an algorithm in the appendix which solves the quadratic problem by solving a sequence of linear integer programming problems. No efficient optimal algorithm is known for the sub-problems (FIX $\langle l, u\rangle$ ). A sub-optimal algorithm is proposed in the appendix but is not evaluated. For the results in this paper, we have solved the sub-problems to optimality with integer programming techniques. 
Based on the bit matrices, the problem instances for the corresponding optimization problems were converted into a linear integer program in standard form using the modeling language ZIMPL [17]. We have solved all resulting integer programs using CPLEX 9.0, a stateof-the-art code for linear and integer programming. The software uses a Branch\&Bound\&Cut algorithm with an implementation of the simplex algorithm for solving linear programming subproblems; outlines of how these algorithms work can be found in [13]. For problems (FIX) and (VAR APP), only linear programming is actually used as the Branch\&Bound Tree collapses to the root node (cf. [16]). The resulting assignments were collected by a script. The script kept record of each frame throughput while running through the complete trace file. Typically, each trace file represented a few seconds (equaling a few thousands of down-link phases). Finally, from the total amount of bits transmitted to each terminal the throughput per terminal was obtained.

\section{B. Scenario Parameterization}

We choose the following basic simulation scenario: The cell radius is $r_{\text {cell }}=100 \mathrm{~m}$, the center frequency is $f_{\mathrm{c}}=5.2 \mathrm{GHz}$. The maximum transmit power equals $P_{\max }=10 \mathrm{~mW}$. The guard interval length is set to $T_{\mathrm{g}}=0.8 \mu \mathrm{s}$ (in correspondence to IEEE 802.11a [18]).

The sub-carrier attenuations $h_{j, n}^{(t)}$ are generated based on the three components path loss, shadowing and fading. For the path loss, a standard model $a_{\mathrm{pl}}^{(t)}=K \cdot \frac{1}{\left(d^{(t)}\right)^{\alpha}}$ is assumed [10]. As parameters, we use $K=46.7 \mathrm{~dB}$ and $\alpha=2.4$, corresponding to a large open space propagation environment. For the shadowing, we assume independent stochastic samples $\left(a_{\mathrm{sh}}^{(t)}\right)$ from a log-normal distribution, characterized by a zero mean and a variance of $\sigma_{\mathrm{sh}}^{2}=5.8 \mathrm{~dB}$. The samples are regenerated every second. While the path loss and shadowing affects all sub-carriers of a terminal alike, each sub-carrier experiences its own fading component. Each sample $a_{\text {fad }}^{(t)}$ of the fading process is assumed to be Rayleigh-distributed. The frequency and time correlation of $a_{\text {fad }}^{(t)}$ are characterized by a Jakes-like power spectrum and an exponential power delay profile. The Jakes-like power spectrum is parameterized by the maximum speed within the propagation environment $v_{\max }$ and the center frequency $f_{\mathrm{c}}$. The exponential power delay profile is characterized by the delay spread $\Delta \sigma=0.15 \mu \mathrm{s}$. The noise power $\sigma^{2}$ is computed at an average temperature of $20^{\circ} \mathrm{C}$ over the bandwidth of a sub-carrier.

For the adaptive modulation scheme, a target symbol error probability of $p_{\text {sym,max }}=10^{-2}$ is chosen for the payload transmission. A total of $M=4$ modulation types are available, namely BPSK, QPSK, 16-QAM and 64-QAM. Thus, BPSK is applied in the SNR range between 4 and 
$9 \mathrm{~dB}$, QPSK is applied between 9 and $16 \mathrm{~dB}, 16-\mathrm{QAM}$ is applied between 16 and $22 \mathrm{~dB}$, and 64-QAM above a SNR value of $22 \mathrm{~dB}$. For the signaling phase, we use BPSK in combination with a rate $1 / 2$ convolutional coder (assuming soft decision), resulting in $b_{\text {sig }}=0.5$. Using our path loss model and considering a $10 \mathrm{~dB}$ fading margin, a SNR of at least $4 \mathrm{~dB}$ is thus reached at the cell border. This yields a bit error probability of $10^{-4}$ [19] in the signaling part; given this small value, we do not consider bit errors in the signaling part ${ }^{5}$.

\section{Parameter Investigation}

Obviously, the impact of the presented signaling models on the throughput of a dynamic OFDM system depends on a set of parameters, i.e. the number of terminals $J$, the number of sub-carriers $N$ and the number of modulation types $M$. These three parameters directly influence the number of bits in the signaling bit-stream, for both the fixed size model and the the variable size model.

In order to identify parameters with high impact, we first performed a sensitivity analysis following the $2^{k}$ factorial design [21]. As metric we considered the average percentage of symbols per down-link phase required to transmit the signaling information $(=\varsigma / S)$. As Table I shows, the number of sub-carriers $N$, the available system bandwidth $B$ and the length of a frame $T_{\mathrm{f}}$ were found to be the parameters with the highest impact on the signaling percentage. For further details, we refer to [20].

These three parameters are investigated below regarding the net throughput. The number of terminals in the cell was fixed to $J=8$ for these investigations. As $J$ might vary drastically over time in a real setting, we have also investigated the performance behavior under a varying number of terminals. Results on this and on other parameter variations are left out here due to space limitations, they can be found in [20].

For the following discussion, all parameters not specifically mentioned have been fixed as introduced in Section V-B. As comparison schemes for the signaling variants we consider the gross throughput of the introduced dynamic OFDMA scheme (representing the performance results if the signaling overhead is neglected) and a static scheme, where each terminal receives

\footnotetext{
${ }^{5}$ Bit errors in the signaling part play an important role for the performance evaluation. For all signaling schemes we developed simple procedures to deal with bit errors such that the performance loss is quite low. Refer to [20] for details.
} 
a fixed block of sub-carriers during all down-link phases ${ }^{6}$.

1) Varying the Number of Sub-carriers $N$ : For any OFDM system applying a guard interval, increasing the number of sub-carriers under fixed total transmit power and total system bandwidth leads to a throughput increase. The higher the number of sub-carriers, the longer is the symbol duration per sub-carrier. Less guard intervals thus occur per time unit, and the utilization of each sub-carrier is increased, leading to a higher gross system throughput. A large number of sub-carriers thus seems favorable if the signaling overhead is neglected ${ }^{7}$. However, the signaling overhead increases, so the net throughput is affected differently.

Initially, we consider a system of bandwidth $B[\mathrm{~Hz}]=16.25 \mathrm{MHz}$. The transmit power is set to $P_{\max }=10 \mathrm{~mW}$ while $v_{\max }$ equals $1 \mathrm{~m} / \mathrm{s}$. A frame length of $T_{\mathrm{f}}=2 \mathrm{~ms}$ is assumed, equally split into up-link and down-link phase.

Figure 3 shows the net throughput while varying the number of sub-carriers for the schemes with and without signaling overhead. As described above, the net throughput curves of both the static comparison scheme and the dynamic OFDMA approach without signaling overhead increase as the number of sub-carriers increases. The increase is slightly steeper for the dynamic approach: Per terminal it amounts to $250 \mathrm{kBit} / \mathrm{s}$ on average compared to $200 \mathrm{kBit} / \mathrm{s}$ for the static scheme.

The advantage of the dynamic approach without signaling overhead over the static approach is at about $600 \mathrm{kBit} / \mathrm{s}$, about $50 \%$ of the net throughput. This performance advantage has to be seen as upper limit which can be achieved by this specific dynamic OFDMA approach.

However, if signaling cost is considered, a different behavior of the system is exposed. For all dynamic schemes with signaling cost, the net throughput first increases up to a maximum point, and decreases for larger sub-carrier numbers again. As indicated above, for large subcarrier numbers the signaling cost consumes more performance than is gained in terms of gross throughput. Not all signaling schemes are affected in the same way. The variable signaling field approaches perform better than the fixed size signaling field approach. At the maximum net throughput point (at about $N=128$ ), the performance advantage per terminal is about $500 \mathrm{kBit} / \mathrm{s}$ for both variable size signaling field approaches, compared to the static scheme. The

\footnotetext{
${ }^{6}$ In all performance studies regarding the static scheme the results are rather optimistic. The static scheme is still assumed to employ adaptive modulation per sub-carrier. Hence, a signaling scheme would be required to indicate the different modulation types used per sub-carrier. However, such a scheme is not considered in our performance results.

${ }^{7} \mathrm{~A}$ higher number of sub-carriers provides also a finer granularity to adjust assignments to current rate requirements. It may also increase the frequency diversity of the system.
} 
fixed size signaling field approach has a slightly lower throughput than the variable size signaling field approaches, the performance advantage over the static approach is about $450 \mathrm{kBit} / \mathrm{s}$. The optimal solution for the variable size signaling field approach achieves at the maximum point only a slightly better result than the approximation. However, for larger sub-carrier numbers the difference between all three signaling approaches becomes more evident. At the highest number of sub-carriers $(N=512)$ the fixed size signaling field approach achieves the same net throughput as the static approach. It is outperformed by the approximation of the variable size signaling field approach (by $150 \mathrm{kBit} / \mathrm{s}$ ) and by the optimal solution to the variable size signaling field model (by $250 \mathrm{kBit} / \mathrm{s}$ ).

As the number of sub-carriers increases, the up-link overhead increases for all five schemes, too. Although our focus is on the down-link explicitly, consider an OFDM-TDMA up-link organization. Per sub-carrier the measured SNR is reported back to the access point using 5 bits (as discussed in Section II). In case of $N=64$ this results in an overhead of 320 bits per terminal. In this case, the up-link consists of 211 OFDM symbols. For each terminal about 25 OFDM symbols remain. Assuming on average a QPSK transmission scheme, this yields a raw up-link capacity per terminal of $25 \cdot 64 \cdot 2=3200$ bits per terminal. Thus, ten percent are lost due to the up-link overhead. In case of $N=512$, per up-link phase 25 OFDM symbols are available in total, resulting in a raw capacity per terminal of $3 \cdot 512 \cdot 2=3072$ bits. However, the feed-back of the sub-carrier attenuations requires now 2560 bits. This equals $83 \%$ of the up-link capacity per terminal. Clearly, as the number of sub-carriers increases, the up-link overhead increases, too. In principle, there exist some options to reduce this overhead. In future work, this issue remains to be investigated in more depth as it is closely related to the channel knowledge issue.

2) Varying the System Bandwidth B: For any given bandwidth, an optimal number of subcarriers can be obtained. Thus, we vary the bandwidth between $10 \mathrm{MHz}$ and $50 \mathrm{MHz}$ and identify for each bandwidth the optimal number of sub-carriers for each scheme (Table II). For the schemes without signaling cost, the preferred sub-carrier number is always the highest possible ${ }^{8}$, independent of the considered bandwidth. For the schemes including signaling cost, the optimal number of sub-carriers increases with bandwidth. The optimal sub-carrier choices regarding the fixed size signaling field model and the approximation of the variable size model are identical. The optimal solution to the variable size signaling field model has higher optimal

\footnotetext{
${ }^{8}$ We varied the sub-carrier numbers between 64 and 1024 .
} 
sub-carrier numbers, as it curbs signaling cost better.

Figure 4 shows the average throughput per terminal for all five schemes at the respective optimal number of sub-carriers of each bandwidth value. Note the linear slope of all five graphs as the bandwidth increases. The gap between the static scheme and the dynamic schemes with signaling increases, as does the gap between the dynamic schemes with signaling and the dynamic scheme without signaling overhead. At their respective optimum values, the different dynamic schemes including the signaling cost differ only marginally. However, note that these throughput values stem from different sub-carrier setting, even for the same bandwidth (cf. Table II).

3) Varying the Frame Length $T_{\mathrm{f}}$ : Next, we vary the length of the frame $T_{\mathrm{f}}$ between $1 \mathrm{~ms}$ and $10 \mathrm{~ms}$. These variations are performed for $N=64$ and $N=512$. The maximum speed within the propagation environment equals $v_{\max }=2 \mathrm{~m} / \mathrm{s}$. The system bandwidth and transmit power are parameterized as with the variation of the number of sub-carriers.

The number of symbols per down-link phase increase with the frame length. A dynamic algorithm will thus reassign sub-carriers less often. However, the sub-carrier attenuations are much more uncorrelated now. This may lead to a higher number of necessary reassignments. The signaling cost may thus explode for the variable size signaling field approaches. In general, a longer frame length is quite attractive from a system point of view: There is more time for the access point to compute new assignments, making the real-time constraint less restrictive. In addition, the signaling cost can be decreased significantly. The downside is the accuracy of the channel knowledge, which will in general degrade the longer the frame length is. A lower accuracy of the channel knowledge might lead to a higher bit error probability during the payload transmission. On the other side, the usage of a longer frame length might enable the usage of sophisticated channel estimation techniques, which would again improve the accuracy of the channel knowledge. This trade-off is not studied here, as we assume the attenuations to be constant during the down-link phase. Note that also regarding the up-link overhead a longer frame length is preferable.

In Figure 5 and 6 the net throughput is given for all five investigated schemes in a setting with $N=64$ sub-carriers and $N=512$ sub-carriers. In both cases, the above mentioned effects can be observed. While an increasing frame length has no effect at all for the static scheme as well as for the dynamic scheme without signaling overhead, a longer frame length leads to a higher net throughput for the dynamic schemes with signaling cost. This effect is much more significant with a high number of sub-carriers. In both cases, the potential benefit from 
using a dynamic approach is about $550 \mathrm{kBit} / \mathrm{s}$ on average per terminal which is about $50 \%$ more throughput per terminal than with the static scheme. If the length of the frame is low, the fixed size signaling field approach yields the lowest net throughput of all dynamic schemes with signaling cost. The optimal solution of the variable size signaling field approach yields the best net throughput in these cases. Note that in case of the low number of sub-carriers the difference between worst and best dynamic scheme with signaling cost is rather small. In case of $N=512$ the net throughput of the fixed size signaling field approach is even lower than the performance of the static scheme.

This performance behavior changes for longer frame lengths. For $T_{\mathrm{f}}$ between $3 \mathrm{~ms}$ and $4 \mathrm{~ms}$ the fixed size signaling field approach performs better in terms of net throughput than the variable size signaling field approaches. This is not very significant in the case of a low number of sub-carriers; the throughput gain in general is quite low for the dynamic schemes as the frame length increases. However, if the system design includes a high number of sub-carriers, the performance difference between fixed size signaling approach and variable size signaling approach is substantial. It amounts to around $150 \mathrm{kBit} / \mathrm{s}$ on average per terminal $-10 \%$ of the average throughput per terminal at no additional cost. Note that although the net throughput increases constantly for the dynamic schemes including the signaling cost, the highest gains are achieved for rather small frame lengths. In general, for a frame length exceeding $5 \mathrm{~ms}$, the additional net throughput gain is no longer significant. Reducing the frame length from $10 \mathrm{~ms}$ down to $5 \mathrm{~ms}$ might therefore suit the time-selective behavior of the channels much better. It leads to less bit errors without causing a dramatic loss of net throughput.

As the frame length increases, the performance loss due to signaling decreases, too. The performance gap between the dynamic scheme without signaling cost and the dynamic schemes with signaling cost depends on other system parameters which influence the signaling cost. For each parameter setting, there exists a specific frame length from which on the performance degrading effect of the signaling becomes less important (here at $T_{\mathrm{f}}=4 \mathrm{~ms}$ ). If the parameters ruling the signaling overhead lead to a high signaling cost per reassignment, the usage of the fixed size signaling field approach outperforms the variable size signaling field approach. If the length of a frame is high, and thus the correlation of the sub-carrier attenuations is low, the variable size signaling field approach is the better choice.

\section{CONCLUSiONS AND Future WORK}


We find that the qualitative and quantitative behavior of the performance of dynamic OFDMA systems changes significantly if the signaling cost is taken into account. However, it is important to stress that despite this overhead the performance of dynamic OFDMA systems remains always superior to the one achieved by static schemes. Therefore, dynamic OFDMA systems remain a viable performance enhancing option for future and existing wireless communication standards.

The choice of the system parameters is important, however. In certain cases, the usage of the dynamic approach becomes impractical because the overhead is too large. These situations are characterized by a low overall system bandwidth and a high signaling cost per single reassignment. For systems with a high bandwidth (as it is expected for $4 \mathrm{G}$ systems), the usage of dynamic OFDMA approaches is thus affected only marginally by the signaling cost.

The usage of techniques reducing the signaling cost can improve the performance even further in certain cases. In this study, we introduced schemes which exploit the correlation in time in order to reduce the signaling overhead. This works well if the correlation in time is high (for example, for a low velocity in the propagation environment). However, one of these schemes adds computational complexity to the optimization problem to solve, which is a potential drawback.

Depending on the situation (mobility in the environment, the number of terminals in the cell, available system bandwidth) there exists a preferred choice of system parameters that can be influenced by the access point. These system parameters include the number of sub-carriers the bandwidth is split into, number of modulation types, and the used signaling scheme. This motivates the usage of adaptive access points which choose a certain set of system parameters depending on the set of external parameters. This might significantly increase the performance of dynamic OFDMA systems.

As open issue is compression of signaling information. In the context of bit-loading, it has been shown [7] that compression schemes can reduce the signaling overhead noticeably. In certain cases, the gap between the dynamic schemes with signaling overhead and the dynamic scheme without signaling cost is quite large. This gap could be reduced by adding a compression scheme to the signaling information. However, it is not clear by how much the signaling overhead transmitted per down-link phase can be compressed. The signaling bit stream differs substantially from the bit-loading scenario as now also the terminal identification has to be transmitted. This might call for the application of different compression schemes. However, any compression scheme will demand computational resources at the access point as well as 
at the terminals, which is not the case for the schemes presented in this paper.

It might also be possible to further reduce the signaling overhead by grouping adjacent sub-carriers. Due to the correlation in frequency, they are likely to be assigned to the same terminal. In combination with the both signaling models, this can lead to a further decrease of the overhead. On the other hand, such a method would reduce the flexibility of the system to provide data rates at a fine granularity.

\section{REFERENCES}

[1] R. van Nee and R. Prasad, OFDM Wireless Multimedia Communications, chapter 9, Artech House, 2000.

[2] J. Bingham, "Multicarrier modulation for data transmission: an idea whose time has come," IEEE Communications Magazine, vol. 28, pp. 5 -14, May 1990.

[3] D. Kivanc, G. Li, and H. Liu, "Computationally efficient bandwidth allocation and power control for OFDMA," IEEE Transactions on Wireless Communications, vol. 2, no. 6, pp. 1150-1158, 2003.

[4] C.Y. Wong, R.S. Cheng, K.B. Letaief, and R. Murch, "Multiuser OFDM with adaptive subcarrier, bit and power allocation," IEEE Journal on Selected Areas of Communications, vol. 17, no. 10, pp. 1747-1758, Oct 1999.

[5] W. Rhee and J. Cioffi, "Increase in capacity of multiuser OFDM system using dynamic subchannel allocation," in Proc. Vehicular Technology Conference (VTC), 2000, pp. 1085 - 1089.

[6] T. Lestable and M. Bartelli, "LZW Adaptive Bit Loading," in Proc. IEEE Internaltional Symposium on Advances in Wireless Communications, September 2002.

[7] H. Nguyen, J. Brouet, V. Kumar, and T. Lestable, "Compression of Associated Signaling for Adaptive MultiCarrier Systems," in Proc. Proc. Vehicular Technology Conference (VTC Spring), May 2004.

[8] J. Gross, I. Paoluzzi, H. Karl, and A. Wolisz, "Throughput study for a dynamic OFDM-FDMA system with inband signaling," in Proc. Vehicular Technology Conference (VTC Spring), Milan, Italy, May 2004.

[9] J. Gross, H. Karl, and A. Wolisz, "Throughput optimization of dynamic OFDM-FDMA systems with inband signaling," in Proc. of 2nd WiOpt'04 (Modeling and Optimization in Mobile, Ad Hoc and Wireless Networks), Cambridge,United Kingdom, Mar. 2004.

[10] J. Cavers, Mobile Channel Characteristics, chapter 1.3, Kluwer Academic, 2000.

[11] H. Yin and H. Liu, "An efficient multiuser loading algorithm for OFDM-based broadband wireless systems," in Proc. of IEEE Globecom, 2000.

[12] J. Gross, J. Klaue, H. Karl, and A. Wolisz, "Cross-layer optimization of OFDM transmission systems for MPEG-4 video streaming," Computer Communications, vol. 27, pp. 1044-1055, 2004.

[13] B. Korte and J. Vygen, Combinatorial Optimization, Springer, 2000.

[14] J. Gross, H. Karl, F. Fitzek, and A. Wolisz, "Comparison of heuristic and optimal subcarrier assignment algorithms," in Proc. Intl.Conf. on Wireless Networks (ICWN), June 2003.

[15] M. Paetzold, Mobile Fading Channels, chapter 4.1, J. Wiley \& Sons, Inc., 2002.

[16] A. Schrijver, Combinatorial Optimization, Springer, 2003.

[17] Thorsten Koch, ZIMPL User Guide 2.02, Zuse Institute Berlin, May 2004. 
[18] IEEE P802.11a/D7.0, Supplement to Standard for Telecommunications and Information Exchange Between Systems - LAN/MAN Specific Requirements - Part 11: Wireless MAC and PHY Specifications: High Speed Physical Layer in the 5-GHz Band, July 1999.

[19] J. Proakis, Digital Communications, McGraw-Hill, 1995.

[20] J. Gross, H. Geerdes, H. Karl, and A. Wolisz, "Performance analysis of dynamic OFDM-FDMA systems with inband signaling," Tech. Rep. TKN-05-001, Telecommunication Networks Group, Technische Universität Berlin, 2005.

[21] Raj Jain, The art of computer systems performance analysis, J. Wiley \& Sons, Inc., 1991.

\section{APPENDIX}

We reformulate optimization problem (VAR OPT) by considering the integer variable $\varsigma$, denoting the number of OFDM symbols required for conveying the signaling information:

$$
\begin{array}{ll}
\max & (S-\varsigma) \cdot \sum_{j, n}\left(b_{j, n}^{(t)} \cdot x_{j, n}^{(t)}\right) \\
\text { s.t. } \quad(1),(3) & \\
& \varsigma \geq \frac{\sum_{j, n} c_{j, n}^{(t)} \cdot x_{j, n}^{(t)}}{N \cdot b_{\text {sig }}} \\
& \varsigma \text { integral. }
\end{array}
$$

(VAR OPT')

Note that any solution with $\varsigma>\left\lceil\sum_{j, n} c_{j, n}^{(t)} \cdot x_{j, n}^{(t)} / N \cdot b_{\text {sig }}\right\rceil$ can be improved by merely reducing the value of sigma without changing the assignment variables. For any optimal solution, equality will therefore hold and we need not introduce the additional constraint $\varsigma<$ $\left(\sum_{j, n} c_{j, n}^{(t)} \cdot x_{j, n}^{(t)} / N \cdot b_{\text {sig }}\right)+1$.

This problem is quadratic as the objective function includes the product of variables. Several options are available to reduce this problem to a linear one. A quadratic programming approach does not apply here since the matrix of coefficients is not positive definite. A possible solution is a reformulation that essentially introduces one integral variable and two constraints for each product of $\varsigma$ with a binary variable. However, in our context there are very few possible values that $\varsigma$ can take. Therefore, enumeration is a viable alternative. To avoid enumeration of all possible values for $\varsigma$, we employ the following scheme. We divide the problem into subproblems by imposing bounds $0 \leq l \leq u \leq C_{\mathrm{sig}} / b_{\mathrm{sig}}$ on the number of symbol times used for signaling:

$$
\max \quad \text { VAR OPT, } \quad \text { s.t. } \quad l \leq \varsigma \leq u \quad \text { (VAR' }\langle l, u\rangle)
$$

We can estimate the value of the optimum and obtain feasible solutions by solving the easier problem (FIX) with some additional constraints, that is,

$$
\max \quad \text { FIX } \quad \text { s.t. } \quad l-1<\sum_{j, n} c_{j, n}^{(t)} \cdot x_{j, n}^{(t)} / N \cdot b_{\text {sig }} \leq u \quad(\operatorname{FIX}\langle l, u\rangle)
$$


and applying the relation $(S-u) \operatorname{FIX}\langle l, u\rangle \leq \operatorname{VAR}^{\prime}\langle l, u\rangle \leq(S-l) \operatorname{FIX}\langle l, u\rangle$. In particular, if $l=u$, we obtain the optimal solution. Our approach works as follows:

1) Obtain a starting value $\varsigma_{0}$ by computing the number of symbol times used for signaling when using the optimal solution of (FIX).

2) Check for better solutions with $\varsigma_{+}=\varsigma_{0}+1,2, \ldots$ symbols spent on signaling by solving (VAR' $\langle l, u\rangle)$ with $l=u=\varsigma_{+}$, stopping as soon as $\left(S-\varsigma_{+}\right) \cdot \operatorname{FIX}\left\langle\varsigma_{+}, C_{\text {sig }} / b_{\text {sig }}\right\rangle$ is less than the currently best solution (all higher values of $\varsigma$ can then be excluded.)

3) Check for better solutions with $\varsigma_{-}=\varsigma_{0}-1,2, \ldots$ bits spent on signaling by solving (VAR' $\langle l, u\rangle$ ) with $l=u=\varsigma_{-}$, stopping as soon as $S \cdot \operatorname{FIX}\left\langle 0, \varsigma_{-}\right\rangle$is less than the currently best solution value. (All lower values of $\varsigma$ can then be excluded.)

Empirically, the optimal solution is always very close to $\varsigma_{0}$ and enumeration of most possible values for $\varsigma$ can be skipped by the above method.

However, the above mentioned solution approach depends on the complexity of problem $(\mathrm{FIX}\langle l, u\rangle)$. We suspect this problem to be $N P$-hard, but we can not prove it at the moment. In the evaluation, this problem has been optimally solved by using CPLEX. However, we also propose a suboptimal algorithm.

The problem $(\operatorname{FIX}\langle l, u\rangle)$ is a type of weighted bipartite matching problem where a certain number of "old" matches has to be kept fixed for the next matching. Only the number is fixed, not which "old" matches have to kept fixed. The set of all "old" matches is given by the assignment matrix $\mathbf{X}^{(t-1)}$. Denote the number of matches to be kept as $\kappa$.

In order to solve this problem, we propose the following. Initially, sort the old matches in $\mathbf{X}^{(t-1)}$ according to their actual bit value in $\mathbf{B}^{(t)}$. From these sorted matches, keep the $\kappa$ largest ones. These assignments are kept fixed. Delete the rows and columns of the $\mathbf{B}^{(t)}$ which are covered by all matches to be kept. For the reduced bit matrix, denoted as $\tilde{\mathbf{B}^{(t)}}$, solve the weighted bipartite matching problem, using the known efficient algorithm based on b-matching in [13]. 


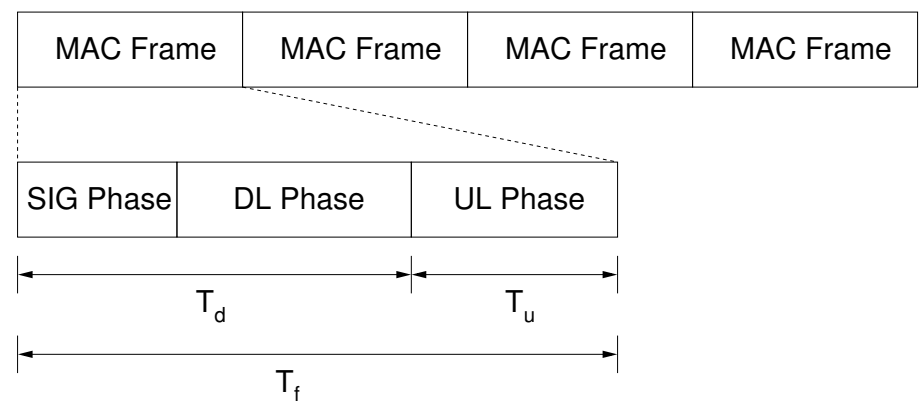

Fig. 1. Basic medium access control layer

\section{Assignment of:}

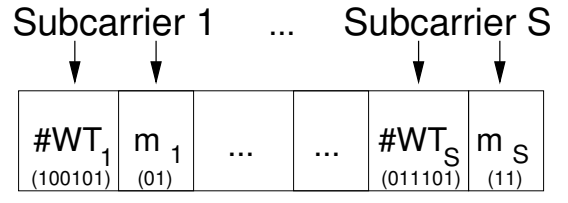

(a) Fixed size signaling field

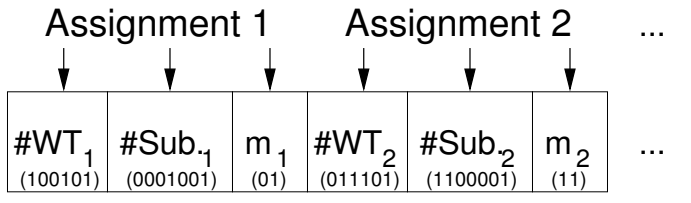

(b) Variable size signaling field

Fig. 2. Representation of assignment information

\begin{tabular}{|c|c|}
\hline Parameter & Average Variation Percentage \\
\hline \hline System bandwidth $B$ & $14.7 \%$ \\
\hline Frame length $T_{\mathrm{f}}$ & $8.4 \%$ \\
\hline Number of sub-carriers $N$ & $7.4 \%$ \\
\hline Number of modulations $M$ & $2.7 \%$ \\
\hline Stochastic variation & $21.8 \%$ \\
\hline$B$ and $T_{\mathrm{f}}$ & $11.4 \%$ \\
\hline$N$ and $T_{\mathrm{f}}$ & $4.7 \%$ \\
\hline$B$ and $N$ & $4.2 \%$ \\
\hline \multicolumn{2}{|c|}{ TABLE I }
\end{tabular}

VARIATION PERCENTAGE OF SELECTED FACTORS AND FACTOR COMBINATIONS REGARDING THE AVERAGE OF THE SIGNALING OVERHEAD FOR THE THREE SIGNALING MODELS INTRODUCED. 


\begin{tabular}{|c|c|c|c|c|c|}
\hline & $B=10 \mathrm{MHz}$ & $B=20 \mathrm{MHz}$ & $B=30 \mathrm{MHz}$ & $B=40 \mathrm{MHz}$ & $B=50 \mathrm{MHz}$ \\
\hline \hline Static & 1024 & 1024 & 1024 & 1024 & 1024 \\
\hline Fixed Sig. Model & 64 & 128 & 256 & 256 & 512 \\
\hline Var. Sig. Model Approx. & 64 & 128 & 256 & 256 & 512 \\
\hline Var. Sig. Model Opt. & 128 & 256 & 256 & 512 & 512 \\
\hline Dynamic No Sig. Cost & 1024 & 1024 & 1024 & 1024 & 1024 \\
\hline
\end{tabular}

OPTIMAL CHOICE OF SUB-CARRIERS FOR ALL STUDIED SCHEMES WHILE VARYING THE BANDWIDTH BETWEEN

$$
B=10 \mathrm{MHz} \text { AND } B=50 \mathrm{MHz} \text {. }
$$

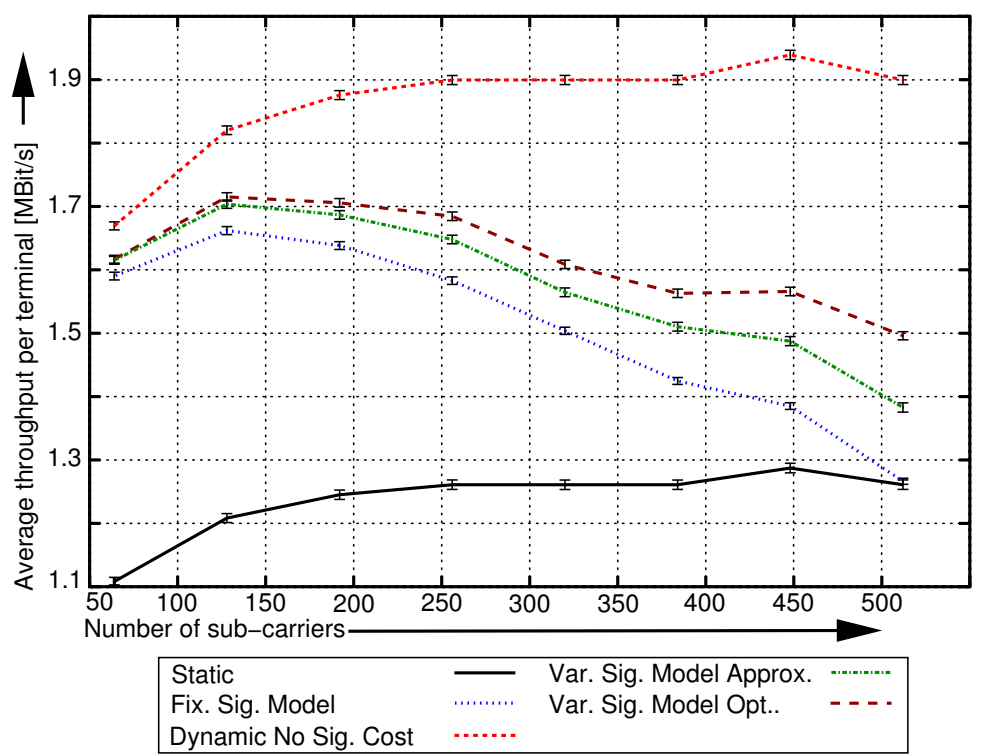

Fig. 3. Average net throughput per terminal for an increasing number of sub-carriers $\left(v_{\max }=1 \mathrm{~m} / \mathrm{s}-\right.$ confidence level 0.99$)$. 


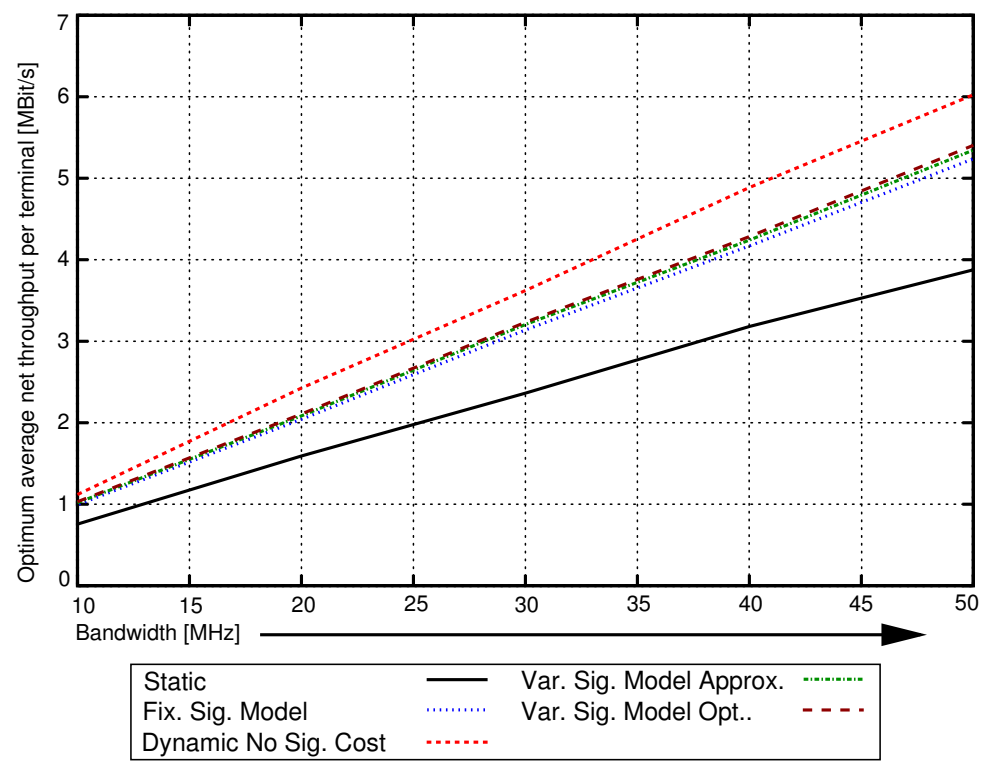

Fig. 4. Optimal net throughput per terminal for an increasing system bandwidth (confidence level 0.99).

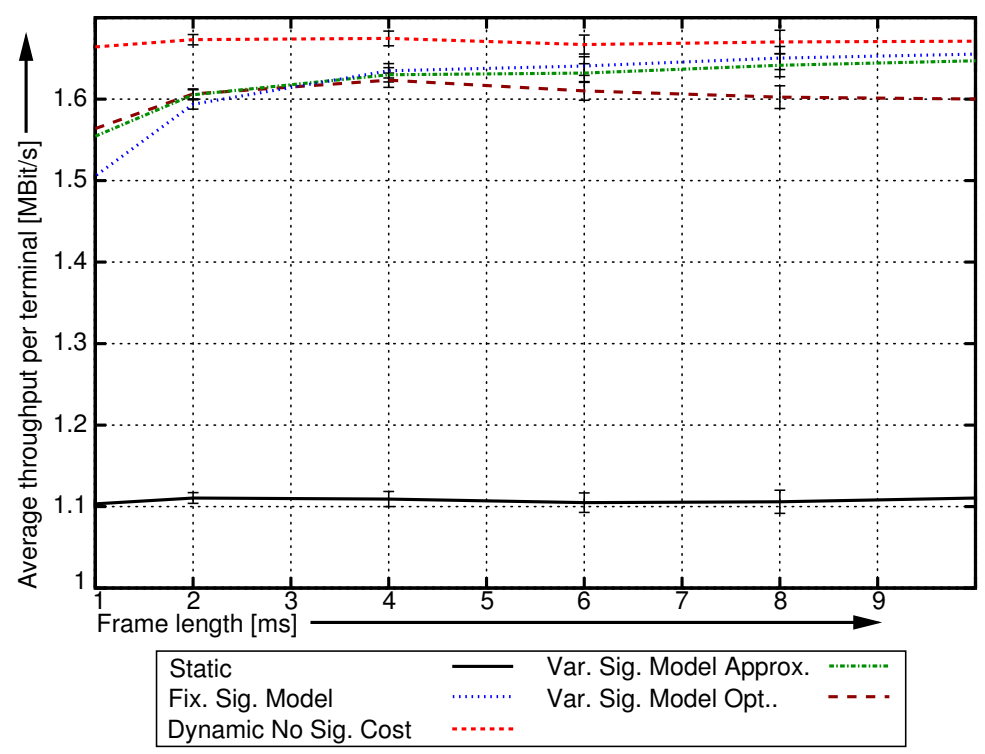

Fig. 5. Average net throughput per terminal for an increasing frame length with $N=64$ sub-carriers (confidence level 0.99 ). 


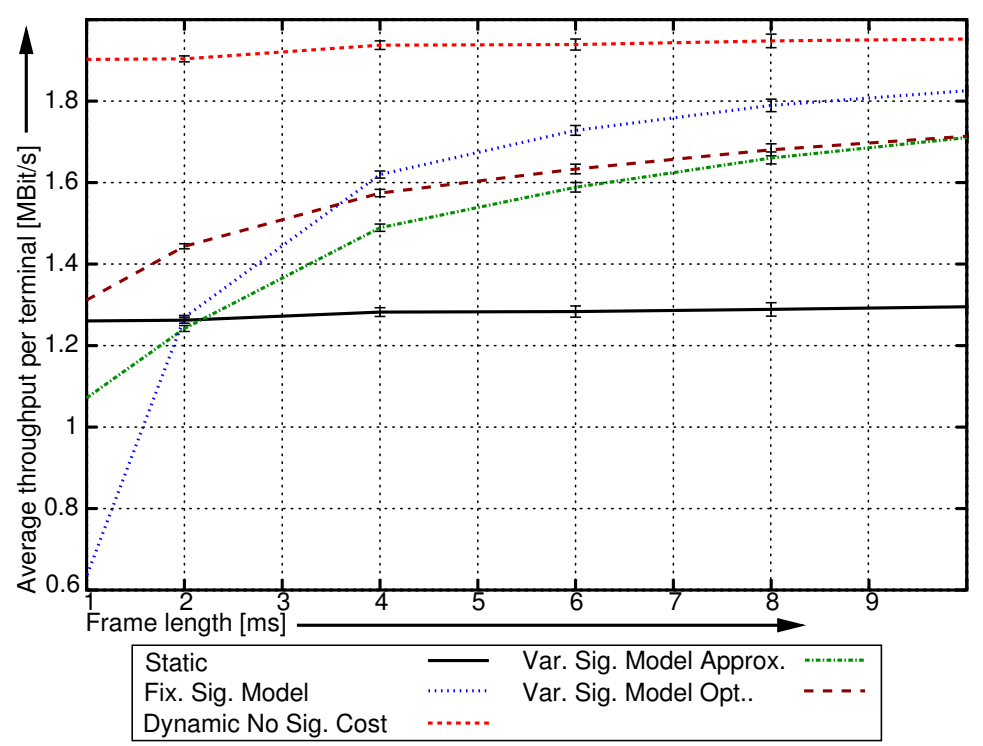

Fig. 6. Average net throughput per terminal for an increasing frame length with $N=512$ sub-carriers (confidence level 0.99 ). 